\title{
Dynamic Locomotion on Slippery Ground
}

\section{Journal Article}

\section{Author(s):}

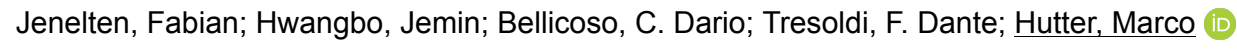

Publication date:

2019-10

Permanent link:

https://doi.org/10.3929/ethz-b-000355281

\section{Rights / license:}

In Copyright - Non-Commercial Use Permitted

\section{Originally published in:}

IEEE Robotics and Automation Letters 4(4), https://doi.org/10.1109/LRA.2019.2931284

\section{Funding acknowledgement:}

780883 - subTerranean Haptic INvestiGator (EC) 


\title{
Dynamic Locomotion on Slippery Ground
}

\author{
Fabian Jenelten ${ }^{1}$, Jemin Hwangbo ${ }^{1}$, Fabian Tresoldi $^{1}$, C. Dario Bellicoso ${ }^{1}$, Marco Hutter ${ }^{1}$
}

\begin{abstract}
Dynamic locomotion on unstructured and uneven terrain is a challenging task in legged robotics. Especially when it comes to slippery ground conditions, common state estimation and control algorithms suffer from the usual noslip assumption. In fact, there has been only little research on this subject. This paper addresses the problem of slipping by treating slip detection and recovery tasks separately. Our contribution to the former is a probabilistic slip estimator based on a Hidden Markov Model. In the second part of this paper, we propose impedance control and friction modulation as useful tools to recover stability during traction loss. We demonstrate the success of our estimation/control architecture by enabling ANYmal, a quadrupedal torque-controllable robot, to dynamically walk over slippery terrain.
\end{abstract}

\section{INTRODUCTION}

The potential of legged robots to assist or replace humans in complex, tedious and dangerous workplaces is tremendous. Inspection, maintenance and search and rescue are particularly interesting fields for legged systems as they are not constrained to flat ground. To fulfill associated mission, it is required that the robot can safely traverse terrains that might be partially slippery. Examples are wet floors in a sewer, dust in a construction site, mud in a mine, oily surfaces on an offshore platform, ice on a street or marble floors.

The slip phenomenon is highly non-linear and difficult to model. It does not come as a surprise that slip-aware controllers have not yet found its way into the legged robotics community. However, the issues related to slippage are critical. As soon as traction is lost, the desired contact force might not be realizable. The limb accelerates along the surface until it can no longer provide support for the torso, and the system unavoidably collapses.

The quadrupedal robot Spot, developed by Boston Dynamics, has shown its remarkable skills while walking over frozen ground, even while being disturbed. ${ }^{1}$ Unfortunately, only little is known about the underlying control system.

An attempt to compensate for traction loss on bipedal robots was shown in [1]. After the horizontal acceleration of a grounded foot reaches a threshold, the robot accelerates the hip in the direction perpendicular to the contact plane. This method is limited to short slip events since the maximum

This research was supported by the Swiss National Science Foundation through the National Centre of Competence in Research Robotics (NCCR Robotics).

This project has received funding from the European Unions Horizon 2020 research and innovation programme under grant agreement No 780883.

${ }^{1}$ All authors are with the Robotic Systems Lab, ETH Zurich, Switzerland ffabian.jenelten, jemin.hwangbo, trfabian, bellicoso\}emavt.ethz.ch

${ }^{1}$ https://www.youtube.com/watch?v=M8YjvHYbZ9w

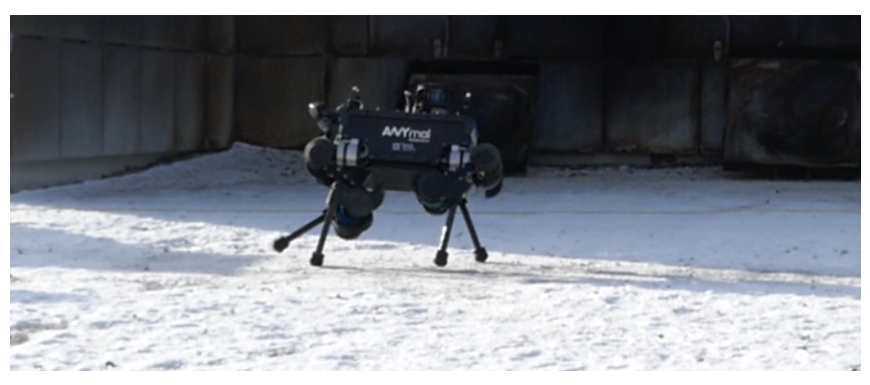

Fig. 1. ANYmal can walk blindly over ice and handle slipping contacts A probabilistic slippage estimator triggers impedance control and friction modulation to stiffen the motion.

attainable torso height is constraint by the kinematics. For the bipedal robot HRP-2 [2], a slip observer based on ground reaction forces has was formulated. A stabilizing reaction was achieved by adjusting the desired footholds to compensate for the torso rotation caused by the sliding motion. The quadrupedal robot TITAN-VIII [3] reacts to slippage, detected in terms of leg-acceleration, by adapting the gait pattern variables. Gait adaptation was shown to be effective, but the long converge time does not guarantee immediate stabilization. To this end, a contact force perturbation perpendicular to the local ground is applied to the supporting foot to potentially push the contact force back into the friction cone. A combination of slip detection and recovery strategy was presented for $H y Q$ [4] and verified in simulation. A velocity-based slip detector triggers a parameter estimator that corrects surface normals and friction coefficients. In [5], a method was shown where contact probabilities are learned from six-dimensional contact-wrench measurements. Slippage was indicated if one or more of the six contact probabilities were significantly lower than the others. Neither slip detection nor recovery was treated in this work. Recent publications dealing with contact estimation are not addressing the slip problem [6], [7] or do not differentiate between slip and swing phases [8] However, slip information can be useful on the locomotion (initiate reactive behavior) and on the state estimation side (avoid drift in the odometry). Mechanical devices s.a. tactile sensors [9], [10] or shear force sensors [11] might be used for slip detection as well. Since these kind of sensors are difficult to maintain on a legged system due to repeated impacts, we do not consider such mechanical mechanisms.

ANYmal (see Fig. 1), a rugged quadrupedal robot developed for outdoor operations, has recently demonstrated the ability to dynamically walk using a variety of gaits [12]. Reference trajectories are generated using a Zero-Moment Point (ZMP) [13] based approach and tracked through a hier- 


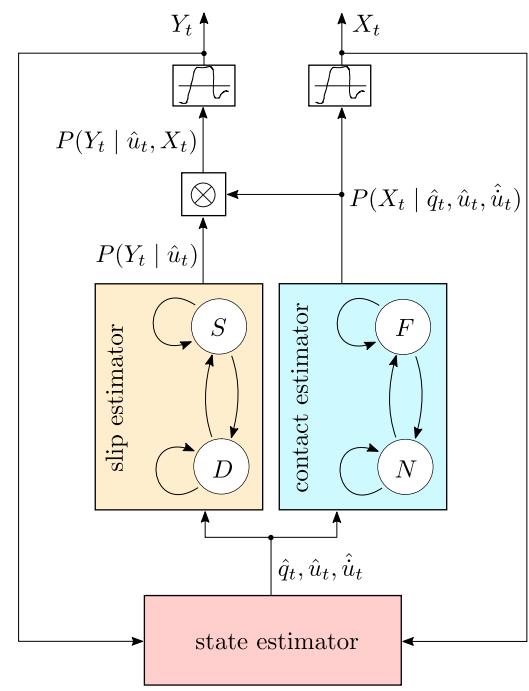

Fig. 2. Combined contact and slip estimation framework. The state estimator provides an estimate of the generalized coordinates $\mathbf{q}$, the velocities $\mathbf{u}$ and the accelerations $\dot{\mathbf{u}}$ using - among other measurements - the contact/slip state of the previous iteration as inputs. Two probabilistic state machines compute for each end-effector the contact probability $P\left(X_{t} \mid \hat{\mathbf{q}}, \hat{\mathbf{u}}, \hat{\mathbf{u}}\right)$ and the slip probability $P\left(Y_{t} \mid \hat{\mathbf{q}}, \hat{\mathbf{u}}, \hat{\dot{\mathbf{u}}}\right)$. Since the state machines are updated independently of each other, a correction step $\otimes$ is required (see (10) and (11)). Contact state $X_{t} \in\{N=$ on, $F=$ off $\}$ and slip state $Y_{t} \in\{S=$ slip, $D=$ ground-fixed $\}$ follow by thresholding the associated probabilities

archical whole-body controller (WBC) [14]. Neither the setpoint generation nor tracking algorithm is aware of slippage, which limits the application to specific terrains.

In the following, we introduce an approach to overcome this limitation. The main contribution is a method for robust slip detection that may be applied to any legged system interacting with the environment through point feet or nonactuated flat feet. End-effectors are modeled as 3 degrees of freedom (DOF) points and both, contact and slip states are considered binary. We farther show how the slip state can be employed in a WBC-based locomotion framework in order to stabilize sliding contacts. Finally, we demonstrate the success of the combined estimation/control pipeline in a series of experiments with ANYmal.

\section{Contact And Slip Estimation}

Since both sliding and stable contacts impose related constraints on the end-effector ${ }^{2}$, it makes sense to combine their estimation in one single framework. We build upon the probabilistic contact estimator presented in [6] and add a probabilistic slip detector in parallel. As depicted in Fig. 2, both estimators are tightly integrated into our state estimation framework [15], which fuses information from leg kinematics and IMU data. The kinematic constraints used in the state estimator rely on accurate contact flags. A slip event is thereby treated as a swing event.

\footnotetext{
${ }^{2}$ For instance, no velocity along the perpendicular direction of the contact plane. Additionally, a stable and a sliding contact can both give sufficient support to stabilize the torso due to non-trivial ground reaction forces.
}

\section{A. Probabilistic Contact Estimation}

We have implemented a probabilistic state machine presented in [6]. To the author's knowledge, it is the first time that this algorithm is used in robotics for contact estimation. For clarification of following formulas, we briefly recapitulate the underlying idea. The model under consideration consists of a measurement model (reflecting the likelihood of observing the robot's state given the contact state) and a transition model (indicating the lift-off or touch-down probability). We will use the notation introduced in [6], i.e., the contact state is denoted by $X_{t} \in\{N, F\}$ where $N$ means on and $F$ off the terrain. The generalized coordinates and velocities of the robot are denoted by $\mathbf{q}$ and $\mathbf{u}$. For their measured or estimated counterpart we write $\hat{\mathbf{q}}$ and $\hat{\mathbf{u}}$.

The measurement model combines information from the dynamics, the differential kinematics, and the kinematics. According to the rule of conditional probability distribution function (PDF), we can write

$$
\begin{aligned}
& P\left(\hat{\mathbf{q}}_{t}, \hat{\mathbf{u}}_{t}, \hat{\dot{\mathbf{u}}}_{t} \mid X_{t}\right)= \\
& \quad \underbrace{P\left(\hat{\mathbf{u}}_{t} \mid X_{t}, \hat{\mathbf{u}}_{t}, \hat{\mathbf{q}}_{t}\right)}_{\text {dynamics }} \cdot \underbrace{P\left(\hat{\mathbf{u}}_{t} \mid X_{t}, \hat{\mathbf{q}}_{t}\right)}_{\text {diff. kinematics }} \cdot \underbrace{P\left(\hat{\mathbf{q}}_{t} \mid X_{t}\right)}_{\text {kinematics }},
\end{aligned}
$$

where $\hat{\mathbf{q}}_{t}=\hat{\mathbf{q}}(t)$ for the sake of brevity. As an example, $P\left(\hat{\mathbf{q}}_{t} \mid X_{t}\right)$ is the probability of observing the robot in the kinematic configuration $\hat{\mathbf{q}}_{t}$ given the contact state $X_{t}$.

The transition model can be expanded using the Bayes theorem as

$$
\begin{aligned}
& P\left(X_{t} \mid X_{t-1}, \hat{\mathbf{q}}_{t-1}, \hat{\mathbf{u}}_{t-1}, \hat{\dot{\mathbf{u}}}_{t-1}\right)= \\
& \frac{P\left(\hat{\mathbf{q}}_{t-1}, \hat{\mathbf{u}}_{t-1}, \hat{\dot{\mathbf{u}}}_{t-1} \mid X_{t}, X_{t-1}\right) \cdot P\left(X_{t} \mid X_{t-1}\right)}{\sum_{X_{t}} P\left(\hat{\mathbf{q}}_{t-1}, \hat{\mathbf{u}}_{t-1}, \hat{\dot{\mathbf{u}}}_{t-1} \mid X_{t}, X_{t-1}\right) \cdot P\left(X_{t} \mid X_{t-1}\right)} .
\end{aligned}
$$

We are seeking for a general formulation that is independent of the locomotion controller. Compared to the original formulation, we have dropped the dependency on the gait. Since we do not have any prior guess, we set $P\left(X_{t} \mid X_{t-1}\right)=$ 0.5 . The measurement and transition models are combined together through a Hidden Markov Model (HMM) of the form

$$
\begin{aligned}
& \alpha\left(X_{t}\right)=P\left(\hat{\mathbf{q}}_{t}, \hat{\mathbf{u}}_{t}, \hat{\mathbf{u}}_{t} \mid X_{t}\right) . \\
& \quad \sum_{X_{t-1}} P\left(X_{t} \mid X_{t-1}, \hat{\mathbf{q}}_{t-1}, \hat{\mathbf{u}}_{t-1}, \hat{\dot{\mathbf{u}}}_{t-1}\right) \alpha\left(X_{t-1}\right) .
\end{aligned}
$$

The contact probability can be obtained as

$$
P\left(X_{t}=N \mid \hat{\mathbf{q}}_{1: t}, \hat{\mathbf{u}}_{1: t}, \hat{\dot{\mathbf{u}}}_{1: t}\right)=\frac{\alpha\left(X_{t}=N\right)}{\sum_{X_{t}} \alpha\left(X_{t}\right)} .
$$

Finally, the contact state can be inferred by thresholding the contact probability

$$
X_{t}=\left\{\begin{array}{ll}
N & P\left(X_{t}=N \mid \hat{\mathbf{q}}_{1: t}, \hat{\mathbf{u}}_{1: t}, \hat{\dot{\mathbf{u}}}_{1: t}\right)>0.5 \\
F & \text { otherwise }
\end{array} .\right.
$$

Hwangbo et al. [6] used a Monte-Carlo sampling approach to compute the transition model. It is computationally expensive and requires the touch-down location to be known a pri- 


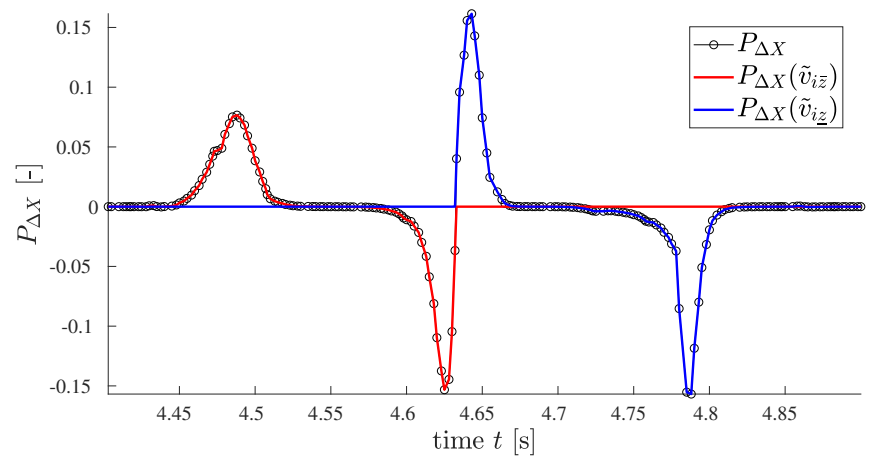

Fig. 3. Signals used for transition indication for contact estimation. The signal $P_{\Delta X}$ has a positive peak at lift-off, two peaks at the height extremum of the swing trajectory, and a negative peak at touch-down. The two peaks corresponding to swing-up can be separated from the swing-down phase by considering only positive (red) or negative (blue) vertical end-effector velocities.

ori, which is not the case for blind locomotion. Therefore, we propose the following lightweight velocity-based approach.

Consider the estimated end-effector velocity $\hat{\mathbf{v}}_{i}^{C}$ of a limb $i$ in control frame $C{ }^{3}$ We consider only the vertical component $\hat{\mathbf{v}}_{i z}^{C}$ and assume that its squared value follows an exponential PDF, i.e., $\operatorname{Exp}\left[\left(\hat{\mathbf{v}}_{i z}^{C}\right)^{2}\right]=\lambda e^{-\lambda\left(\hat{\mathbf{v}}_{i z}^{C}\right)^{2}}$ with $\lambda$ a tuning parameter.

Let $\operatorname{Cexp}[x]=1-e^{-\lambda x}$ be the cumulative distribution function (CDF) of $\operatorname{Exp}[x]$. If the beginning (lift-off) and the end (touch-down) of a swing phase happen at a non-trivial acceleration, then $\operatorname{Exp}\left[\left(\hat{\mathbf{v}}_{i z}^{C}\right)^{2}\right]$ will rapidly change at such an event. This observation leads to the idea of using

$$
P_{\Delta X}=\operatorname{Cexp}\left[\left(\hat{\mathbf{v}}_{i z, t}^{C}\right)^{2}\right]-\operatorname{Cexp}\left[\left(\hat{\mathbf{v}}_{i z, t-1}^{C}\right)^{2}\right]
$$

as a transition indication. Figure 3 shows such a signal recorded in simulation for a trotting gait 4(d). We need to eliminate the two peaks at the height extremum of the swing leg motion and associate the two remaining peaks to the correct event. To do so, we write $\hat{\mathbf{v}}_{i z}^{C}=\tilde{v}_{i \bar{z}}+\tilde{v}_{i \underline{z}}$ with $\tilde{v}_{i \bar{z}} \equiv \max \left\{\hat{\mathbf{v}}_{i z}^{C}, 0\right\}$ and $\tilde{v}_{i \underline{z}} \equiv \min \left\{\hat{\mathbf{v}}_{i z}^{C}, 0\right\}$, and

$$
\begin{aligned}
& P\left(\hat{\mathbf{u}}_{t-1} \mid X_{t}=N, X_{t-1}=F\right)= \\
& -\min \left\{\operatorname{Cexp}\left[\tilde{v}_{i \underline{z}, t}^{2}\right]-\operatorname{Cexp}\left[\tilde{v}_{i \underline{z}, t-1}^{2}\right], 0\right\} \\
& P\left(\hat{\mathbf{u}}_{t-1} \mid X_{t}=F, X_{t-1}=N\right)= \\
& \max \left\{\operatorname{Cexp}\left[\tilde{v}_{i \bar{z}, t}^{2}\right]-\operatorname{Cexp}\left[\tilde{v}_{i \bar{z}, t-1}^{2}\right], 0\right\} .
\end{aligned}
$$

The measurement models as proposed in [6] are further adapted to flat but tilted terrain by performing all Cartesian space computations in control frame rather than in world frame.

\section{B. Probabilistic Slip Estimation}

Similar to the contact state, we define a slip state $Y_{t} \in$ $\{S, D\}$ with $S$ for slipping and $D$ for ground-fixed. The state machine is once again described as a HMM, but this

\footnotetext{
${ }^{3}$ The control frame $C$ is aligned with the local terrain orientation and with the heading direction of the robot. Terrain orientation is obtained by fitting a plane through the recent contact points [16].
}

time, we only take into account the differential kinematics. For a dynamic description of a slip event, we would need an accurate estimate of the friction coefficient. A kinematic description is also difficult to realize as the location, where the slip will end is difficult to predict. The HMM stated in (3) simplifies to

$$
\begin{aligned}
& \beta\left(Y_{t}\right)=P\left(\hat{\mathbf{u}}_{t} \mid Y_{t}, X_{t}\right) \\
& \sum_{Y_{t-1}} P\left(Y_{t} \mid Y_{t-1}, \hat{\mathbf{u}}_{t-1}\right) \beta\left(Y_{t-1}\right) .
\end{aligned}
$$

The measurement model $P\left(\hat{\mathbf{u}}_{t} \mid Y_{t}\right)$, which will be introduced later, can be excited during swing phases and thereby force the slip probability to grow. Since a foot can only slip while it is grounded, we introduce the following heuristic correction

$$
\begin{aligned}
& P\left(\hat{\mathbf{u}}_{t} \mid Y_{t}=S, X_{t}\right):=P\left(\hat{\mathbf{u}}_{t} \mid Y_{t}=S\right) . \\
& P\left(X_{t}=N \mid \hat{\mathbf{q}}_{1: t}, \hat{\mathbf{u}}_{1: t}, \hat{\dot{\mathbf{u}}}_{1: t}\right) \\
& P\left(\hat{\mathbf{u}}_{t} \mid Y_{t}=D, X_{t}\right):=P\left(\hat{\mathbf{u}}_{t} \mid Y_{t}=D\right) \text {. } \\
& P\left(X_{t}=N \mid \hat{\mathbf{q}}_{1: t}, \hat{\mathbf{u}}_{1: t}, \hat{\dot{\mathbf{u}}}_{1: t}\right) \\
& +P\left(X_{t}=F, \mid \hat{\mathbf{q}}_{1: t}, \hat{\mathbf{u}}_{1: t}, \hat{\dot{\mathbf{u}}}_{1: t}\right) .
\end{aligned}
$$

Similar to (2), the transition model is written as

$$
P\left(Y_{t} \mid Y_{t-1}, \hat{\mathbf{u}}_{t-1}\right)=\frac{P\left(\hat{\mathbf{u}}_{t-1} \mid Y_{t}, Y_{t-1}\right)}{\sum_{Y_{t}} P\left(\hat{\mathbf{u}}_{t-1} \mid Y_{t}, Y_{t-1}\right)} .
$$

The probability of slipping can be computed through marginalization as

$$
P\left(Y_{t}=S \mid \hat{\mathbf{u}}_{1: t}, X_{t}\right)=\frac{\beta\left(Y_{t}=S\right)}{\sum_{Y_{t}} \beta\left(Y_{t}\right)},
$$

and thresholding leads to the slip state.

As an indicator of sliding motion we propose a squared tangential foot velocity, i.e., $\tilde{v}_{i m}^{2}=\left(\hat{\mathbf{v}}_{i x}^{C}\right)^{2}+\left(\hat{\mathbf{v}}_{i y}^{C}\right)^{2}$. We assume that the measurement noise of estimate $\hat{\mathbf{v}}_{i}^{C}$ is zeromean Gaussian distributed and the noise is described by the variance $\operatorname{Var}\left[\hat{\mathbf{v}}_{i}^{C}\right]=\operatorname{Diag}\left[\sigma_{v}^{2}\right]$ with Diag being a diagonal matrix. Since $\hat{\mathbf{v}}_{i x}^{C}$ and $\hat{\mathbf{v}}_{i y}^{C}$ are by assumption independent, $\tilde{v}_{i m}^{2}$ follows an exponential PDF $\operatorname{Exp}\left[\tilde{v}_{i m}^{2}\right]=\lambda e^{-\lambda \tilde{v}_{i m}^{2}}$ with gain $\lambda=\frac{1}{2 \sigma_{v}^{2}}$. The associated CDF is used to formulate the measurement model,

$$
\begin{aligned}
P\left(\mathbf{u} \mid Y_{t}=S\right) & =\operatorname{Cexp}\left[\tilde{v}_{i m}^{2}\right] \\
P\left(\mathbf{u} \mid Y_{t}=D\right) & =1-\operatorname{Cexp}\left[\tilde{v}_{i m}^{2}\right] .
\end{aligned}
$$

Assuming a slip-stick phenomenon, a slip event will be initiated at high acceleration and stops at high acceleration. In this case, the signal

$$
P_{\Delta Y}=\operatorname{Cexp}\left[\tilde{v}_{i m, t}^{2}\right]-\operatorname{Cexp}\left[\tilde{v}_{i m, t-1}^{2}\right]
$$

will exhibit two peaks during a slip event, one corresponding to a stick-to-slip and one to slip-to-stick event. We can separate these two by writing

$$
\begin{aligned}
& P\left(\dot{\hat{\mathbf{q}}}_{t-1} \mid Y_{t}=S, Y_{t-1}=D\right)=\max \left\{P_{\Delta Y}, 0\right\} \\
& P\left(\dot{\hat{\mathbf{q}}}_{t-1} \mid Y_{t}=D, Y_{t-1}=S\right)=-\min \left\{P_{\Delta Y}, 0\right\} .
\end{aligned}
$$




\section{Motion Planning And CONTROL}

We use a ZMP-based motion planner to compute the desired torso trajectory as described in [12]. A WBC [14], [17] converts the planned torso and swing-leg trajectories to joint torques by solving a cascade of prioritized tasks. The output of the WBC is a vector of joint torques $\tau_{\text {ref,wbc }} \in \mathbb{R}^{n}$ with $n$ being the number of DOF.

In order to improve the overall tracking in operational space as well as to avoid sudden movements and loss of stability during slippage, we introduce joint-level impedance control. Let $\mathbf{K}_{p}, \mathbf{K}_{d} \in \mathbb{R}^{n \times n}$ be diagonal positive-definite matrices and let $\mathbf{q}_{a}, \mathbf{u}_{a} \in \mathbb{R}^{n}$ denote the generalized coordinates and velocities of the actuated DOF, then

$$
\boldsymbol{\tau}_{\text {ref }}=\boldsymbol{\tau}_{\text {ref,wbc }}+\mathbf{K}_{p}\left(\mathbf{q}_{a, \text { ref }}-\hat{\mathbf{q}}_{a}\right)+\mathbf{K}_{d}\left(\mathbf{u}_{a, \text { ref }}-\hat{\mathbf{u}}_{a}\right) .
$$

The matrices $\mathbf{K}_{p}, \mathbf{K}_{d}$ serve as tuning parameters that are scheduled w.r.t. the ground reaction state $\left\{X_{t}, Y_{t}\right\}$, i.e.,

$$
\mathbf{K}_{p / v}=\left\{\begin{array}{ll}
\mathbf{K}_{p / v, \text { contact }} & X_{t}=N, Y_{t}=D \\
\mathbf{K}_{p / v, \text { swing }} & X_{t}=F \\
\mathbf{K}_{p / v, \text { slip }} & X_{t}=N, Y_{t}=S
\end{array} .\right.
$$

Given a desired state of the end-effector $i$ in Cartesian space $\left\{\mathbf{p}_{i, \text { ref }}, \mathbf{v}_{i, \text { ref }}\right\}$, we obtain joint positions $\mathbf{q}_{a \text {,ref }}$ and velocities $\mathbf{u}_{a \text {,ref }}$ through inverse kinematics. In the following, we describe our approach to find the desired end-effector state depending on the ground reaction state.

\section{A. Swing Leg}

A swing trajectory, obtained as a composition of splines fitted through the previous stance foot position and a desired foothold [16], [18], defines the desired motion of a swinging foot. Trajectory sampling delivers a reference state for the WBC and the impedance controller.

\section{B. Stance Leg}

Assuming a stable contact, we can set $\mathbf{p}_{i \text {,ref }}=\hat{\mathbf{p}}_{i}$ and $\mathbf{v}_{i, \text { ref }}=\mathbf{0}$ with $\hat{\mathbf{p}}_{i}$ the estimated end-effector position.

\section{Slip Leg}

In case a leg is slipping, we want the end-effector to approach towards to the position $\hat{\mathbf{p}}_{i 0}$ where traction was lost. We propose an iterative approach

$$
\begin{aligned}
\hat{\mathbf{p}}_{s} & =\frac{\hat{\mathbf{p}}_{i 0}-\hat{\mathbf{p}}_{i}}{\left\|\hat{\mathbf{p}}_{i 0}-\hat{\mathbf{p}}_{i}\right\|_{2}} \\
\mathbf{v}_{i, \text { ref }} & =\hat{\mathbf{p}}_{s} \cdot \kappa, \quad \mathbf{p}_{i, \text { ref }}=\hat{\mathbf{p}}_{i}+\mathbf{v}_{i, \text { ref }} \cdot T_{s}
\end{aligned}
$$

with $\hat{\mathbf{p}}_{s}$ the sliding vector, $\kappa>0$ a tuning parameter and $T_{s}$ the sampling time of control system. We suggest to chose the impedance gains according to the rule $\mathbf{K}_{p / v \text {,slip }} \gg$ $\mathbf{K}_{p / v \text {,stance }}>\mathbf{K}_{p / v \text {,swing }}$.

During a slip event of leg $i$, we decrease the friction coefficient used in the WBC for leg $i$ to half of its default value. It is reset to the default as soon as the leg has spent more than $0.5 \mathrm{~s}$ in a stable contact.

The impedance control stiffens the joints and thus prevents the slipping leg to diverge, while the friction modulation

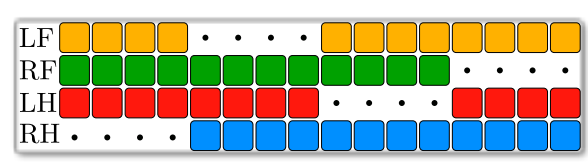

(a) Crawl

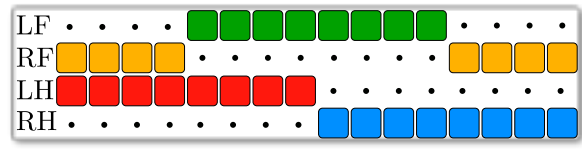

(b) Dynamic Lateral Walk

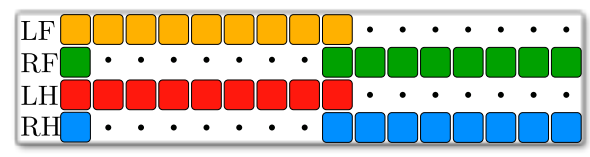

(c) Pace

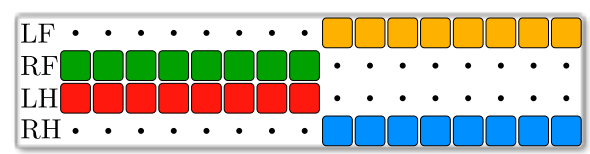

(d) Trot

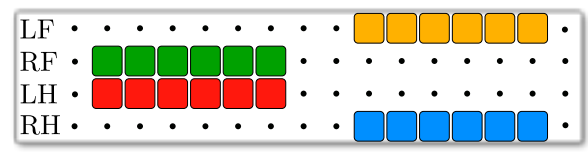

(e) Running trot

Fig. 4. Phase domain description of the gait patterns used in our experiments. Swing phases are indicated with a dot while stance phases are indicated with a colored box. The acronyms represent individual legs: $\mathrm{LF}$ for left front, RF for right front, $\mathrm{LH}$ for left hind and $\mathrm{RH}$ for right hind.

reduces the demanded traction. Notice that, unless the shrunk friction coefficient is not smaller than the real one, the robot is allowed to slip. Also notice that no further adjustments are required to the WBC. In particular, this means that the tracking controller internally does not distinguish between a stable and a sliding contact.

\section{EXPERIMENTS}

To verify our approach, we have conducted several experiments on ANYmal [19], a fully torque-controllable quadrupedal robot. State estimation, contact/slip detection and control run together on an on-board computer (Intel i7$7600 \mathrm{U}, 2.7 \ldots 3.5 \mathrm{Ghz}$, dual-core 64-bit) and are updated at a frequency of $400 \mathrm{~Hz}$. The contact/slip estimation introduces significant overhead in terms of computation: the update step takes roughly $0.11 \mathrm{~ms}$. However, it still meets our real-time requirements.

For all gaits used in the following experiments, the same probability distributions and tuning weights have been used.

\section{A. Contact Estimation}

We have collected data sets of ANYmal walking on flat and non-slippery ground (lab conditions) at an average velocity of $0.3 \mathrm{~m} / \mathrm{s}$. In a first experiment, we used a crawl 4(a). The difficulty associated with this gait is the low 

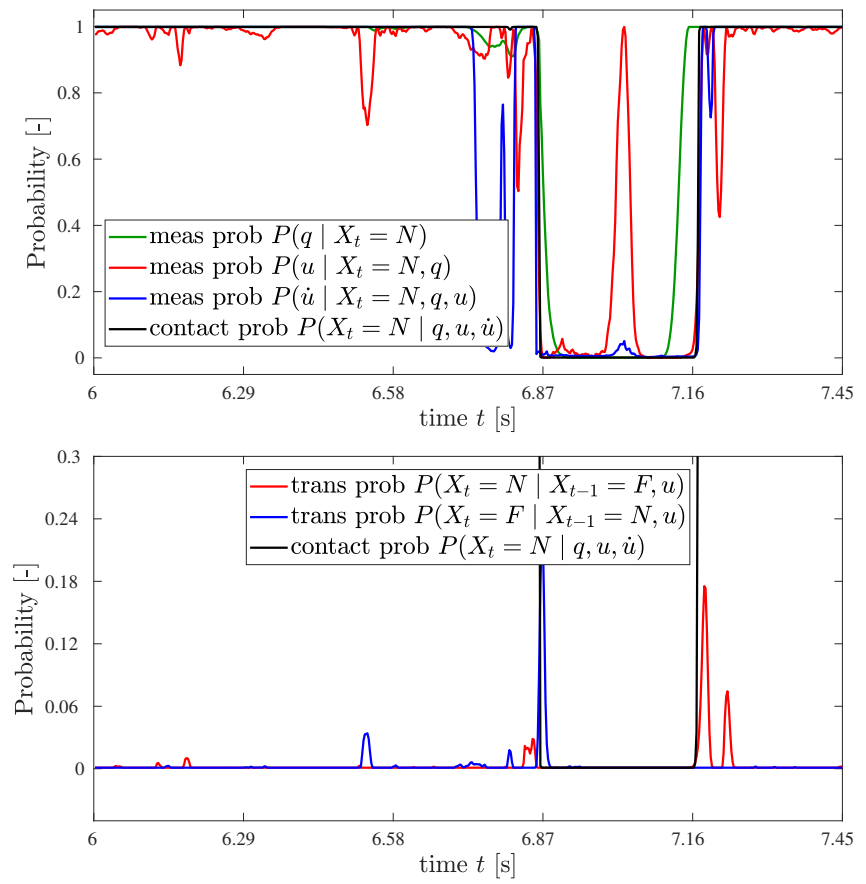

Fig. 5. Contact estimation for the left-front foot of ANYmal while crawling over flat and non-slippery ground. The top plot shows the measurement model and the bottom plot shows the transition model. Shortly before the leg lifts off, the exerted load on that leg is small as the torso leans to the right side. The touch-down event of the left-hind leg further reduces the load which explains the spikes in the dynamics model.

loading factor of a leg shortly before lift-off. In our previous method, the contact state was obtained by thresholding the norm of the ground-reaction force. Such a purely force-based approach may indicate a loss of contact during a stance phase. Consequently, the leg reestablishes the contact by pushing towards the ground. This behavior may cause highfrequency vibrations as the re-bouncing effect can trigger an oscillation. The fusion of three different measurement models robustifies the estimations and eliminates high-frequency contact switches (see Fig. 5).

Another interesting gait is running trot 4(e), because impact forces are comparably large, leading to a re-bouncing effect of the end-effectors during touch-down. As Fig. 6 demonstrates, a contact is established only once at touchdown, and the re-bouncing does not trigger a loss in contact.

In general, the probabilistic approach detects a switch in the ground reaction state at roughly the same speed as our previous contact detector. However, we have observed a significant improvement regarding false detected contact switches. ${ }^{4}$

\section{B. Slip Estimation on high friction Ground}

During a stable contact, it is important that the joints are not unnecessarily stiffened. Otherwise the tracking will be no more optimal in the sense of the cost function used in

\footnotetext{
${ }^{4}$ Our previous estimator detected typically two consecutive contacts at the height extremum of the swing phase.
}
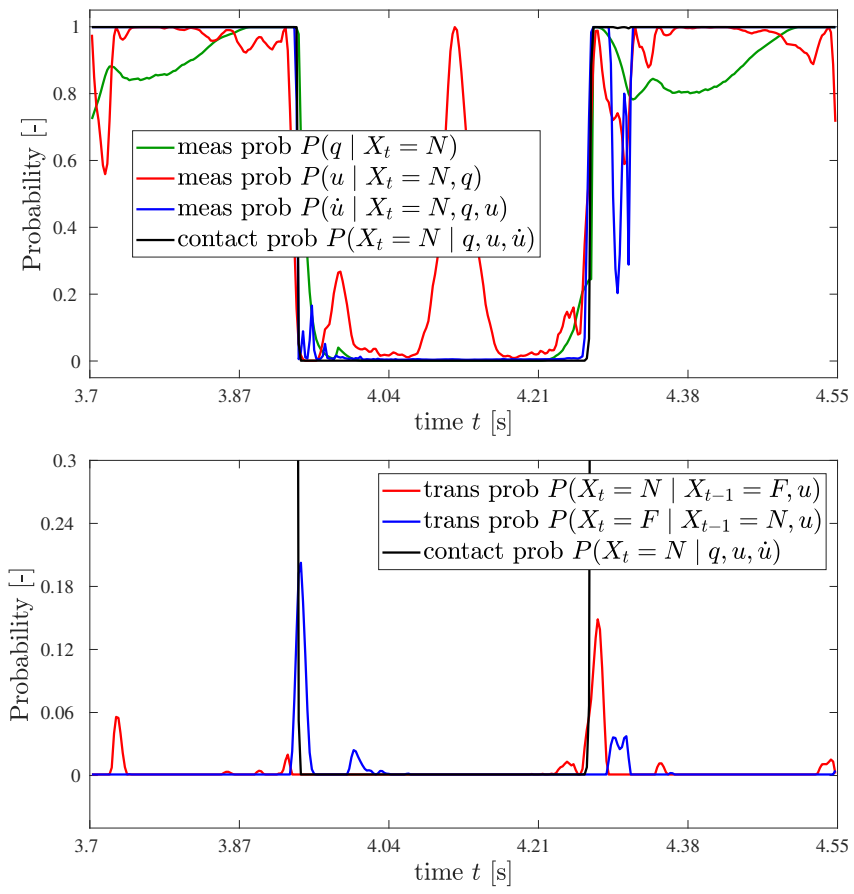

Fig. 6. Contact estimation for the left-front foot of ANYmal while running over flat and non-slippery ground. The top plot shows the measurement model and the bottom plot the transition model. A re-bouncing effect after touch-down can be observed for all three measurement models.

TABLE I

FALSE DETECTED SLIP MEASURED AS (1) TIME SPEND IN SLIP AND (2) NUMBER OF COUNTED SLIP EVENTS.

\begin{tabular}{lll}
\hline Gait & $\begin{array}{l}\text { slip-time per } \\
\text { leg }[\%]\end{array}$ & $\begin{array}{l}\text { peaks/second } \\
\text { per leg }[-]\end{array}$ \\
\hline trot, crawl & 0.0 & 0.0 \\
running trot & 0.34 & 0.24 \\
dynamic lateral walk & 0.01 & 0.13 \\
\hline
\end{tabular}

the WBC. Therefore, slippage should only be detected if the end-effectors do indeed slide.

To verify the robustness against false slip detection, we set up the following experiment: An operator navigates ANYmal through a predefined path on flat and non-slippery ground. The path includes forward, backward and yawing motion at different speeds. We assume that all detected slip events are false positives. Table I presents the results for four different gaits. False detected slip events are due to deformation of the end-effector at touch-down (running trot) or due to vibrations caused by a low loading factor of a leg (dynamic lateral walk, 4(b)).

\section{Slip Estimation on low friction Ground}

A reaction to slip can only occur if the event is also detected as such. Accurate and fast estimation is thus a crucial task for blind locomotion. The experimental set-up used for repeatable slip excitation consists of a whiteboard moistened uniformly with water. In a first experiment, ANYmal is placed on the whiteboard, and the left-front leg is manually displaced (see Fig. 7). 


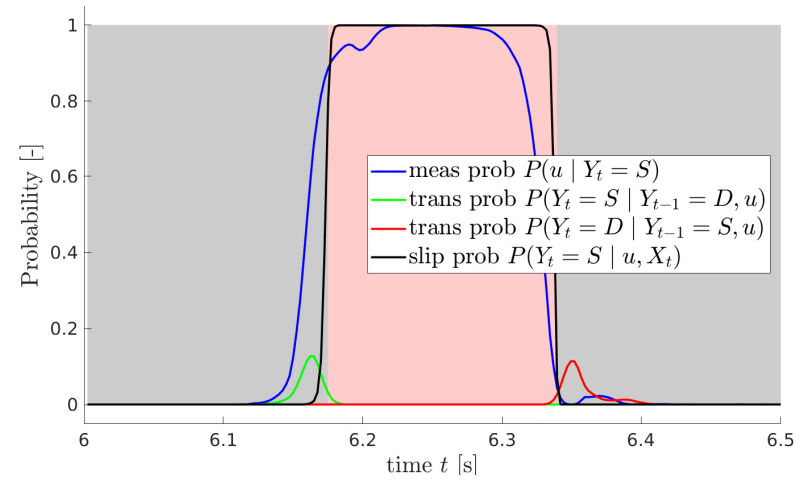

Fig. 7. Slip estimation for the left-front end-effector of ANYmal during manual displacement of that leg. The transition model triggers the slip probability to grow in the beginning and to decay at the end of the applied disturbance. Contact is indicated with a gray background while slippage occurs in the red shaded area.

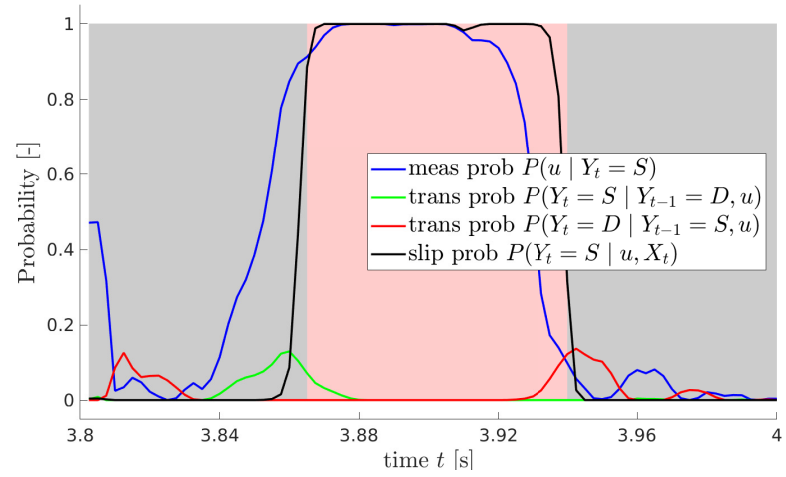

Fig. 8. Slip estimation for the left-front end-effector of ANYmal while pacing over a wet whiteboard. The contact is stable if the background is gray and sliding if it is red.

In a second experiment, our robot walks over the whiteboard using a pacing gate $4(\mathrm{c})$. The recorded slip probabilities are depicted in Fig. 8.

Since it is very difficult to get the actual ground truth of the ground reaction state, validation is shown in terms of control robustification. In order to compare the proposed method to our old configuration, we let ANYmal trot in place with a fixed yawing velocity on top of the wet whiteboard. The experiment was repeated five times per configuration. According to table II, ANYmal could walk three times longer before falling, if the slip compensation was enabled. Moreover, the average time spent in a slip state was reduced by almost a factor of four.

TABLE II

CONTROL PERFORMANCE ON SLIPPERY GROUND USING A TROT.

\begin{tabular}{lll}
\hline slip compensation & slip-time per leg [\%] & time until collapse $[s]$ \\
\hline yes & 0.88 & 69.8 \\
no & 3.20 & 24.1 \\
\hline
\end{tabular}

Our locomotion pipeline has been extensively tested in real-world conditions. ANYmal has recently shown to walk robustly over coarse graveled hills ${ }^{5}$ and has thereby demonstrated to successfully handle slippage similar effects. These effects occurred due to the local displacement of the gravel in the vicinity of the touch-down locations.

In Fig. 9 we show ANYmal recovering from falling after slipping on uneven and frozen ground. A small section of recorded data is displayed in figure 10.

\section{CONCLUSION}

We developed a probabilistic approach for contact and slip estimation, based on a HMM introduced in [6]. The method was extensively tested under laboratory conditions and in field experiments where we demonstrated very reliable detection of the ground reaction state. A slip recovery approach has been presented that relies on invasive impedance control and friction modulation. Field tests on a frozen ground have verified that the presented pipeline can successfully stabilize ANYmal while losing traction.

For the future, we plan to incorporate dynamics and kinematics measurement models to robustify the slip estimation. Next, we can fuse the probabilistic contact and slip estimator into one single state machine. This would allow direct transitions from swing to slip.

\section{REFERENCES}

[1] J. H. Park and O. Kwon, "Reflex control of biped robot locomotion on a slippery surface," in Proceedings 2001 ICRA. IEEE International Conference on Robotics and Automation (Cat. No.01CH37164), vol. 4, May 2001, pp. 4134-4139 vol.4.

[2] K. Kaneko, F. Kanehiro, S. Kajita, M. Morisawa, K. Fujiwara, K. Harada, and H. Hirukawa, "Slip observer for walking on a low friction floor," in 2005 IEEE/RSJ International Conference on Intelligent Robots and Systems, Aug 2005, pp. 634-640.

[3] H. Takemura, M. Deguchi, J. Ueda, Y. Matsumoto, and T. Ogasawara, "Slip-adaptive walk of quadruped robot," Robotics and Autonomous Systems, vol. 53, pp. 124-141, 112005.

[4] M. Focchi, V. Barasuol, M. Frigerio, D. G. Caldwell, and C. Semini, Slip Detection and Recovery for Quadruped Robots. Cham: Springer International Publishing, 2018, pp. 185-199.

[5] N. Rotella, S. Schaal, and L. Righetti, "Unsupervised contact learning for humanoid estimation and control," in 2018 IEEE International Conference on Robotics and Automation (ICRA), May 2018, pp. 411417.

[6] J. Hwangbo, C. D. Bellicoso, P. Fankhauser, and M. Huttery, "Probabilistic foot contact estimation by fusing information from dynamics and differential/forward kinematics," in 2016 IEEE/RSJ International Conference on Intelligent Robots and Systems (IROS), Oct 2016, pp. 3872-3878.

[7] G. Bledt, P. M. Wensing, S. Ingersoll, and S. Kim, "Contact model fusion for event-based locomotion in unstructured terrains," in 2018 IEEE International Conference on Robotics and Automation (ICRA), May 2018, pp. 1-8.

[8] M. Camurri, M. Fallon, S. Bazeille, A. Radulescu, V. Barasuol, D. G. Caldwell, and C. Semini, "Probabilistic contact estimation and impact detection for state estimation of quadruped robots," IEEE Robotics and Automation Letters, vol. 2, no. 2, pp. 1023-1030, April 2017.

[9] A. Narendiran and B. George, "Capacitive tactile sensor with slip detection capabilities for robotic applications," in 2015 IEEE International Instrumentation and Measurement Technology Conference (I2MTC) Proceedings, May 2015, pp. 464-469.

[10] S. Teshigawara, S. Shimizu, K. Tadakuma, M. Aiguo, M. Shimojo, and M. Ishikawa, "High sensitivity slip sensor using pressure conductive rubber," in SENSORS, 2009 IEEE, Oct 2009, pp. 988-991.

${ }^{5}$ https://www.youtube.com/watch?v=7xdk448TRB0\&t=125s 

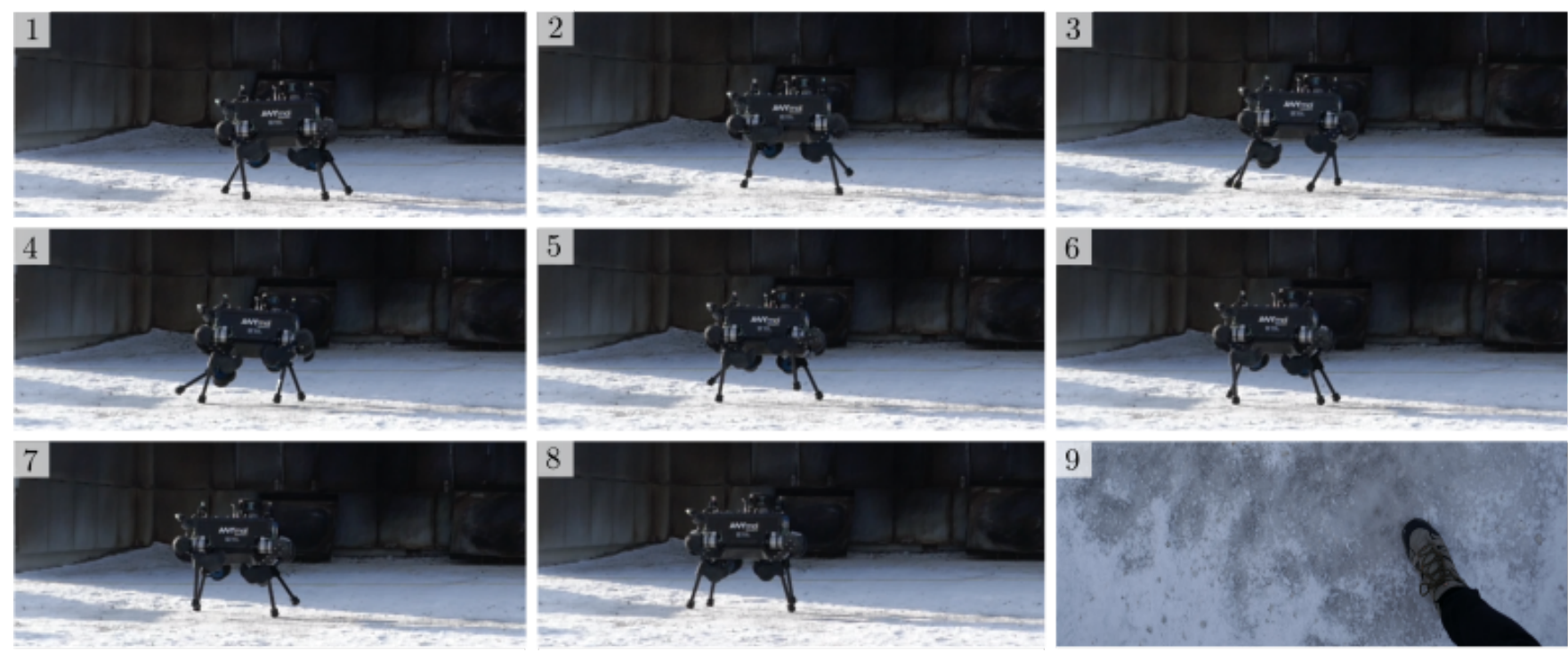

Fig. 9. Impedance control on joint position and velocity level allow ANYmal to walk over slippery ground. As soon as slipping is detected, the robot recovers by stiffen the legs and shrinking the friction cones. The robot's walking direction is from right to left. The bottom right picture (9) shows the terrain passed by the robot.

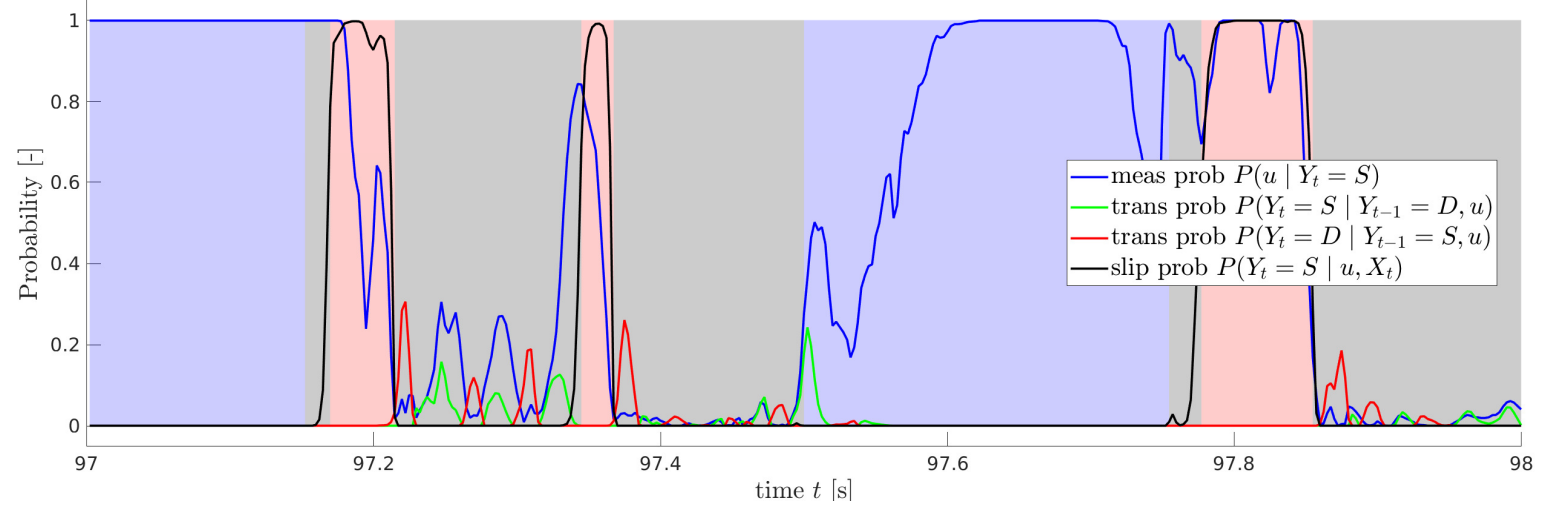

Fig. 10. Slip estimation for the left-front end-effector of ANYmal while trotting over ice. Contact is indicated with a gray background while swing is indicated with a blue and slip with a red shaded background. A slip state can directly switch to a swing state. However, one drawback of the heuristics (10), (11) is the fact that a swing state first has to transit to a stance state before a slip can be detected.

[11] T. Iwasaki, T. Takeshita, Y. Arinaga, K. Uemura, H. Ando, S. Takeuchi, M. Furue, E. Higurashi, and R. Sawada, "Shearing force measurement device with a built-in integrated micro displacement sensor," Sensors and Actuators A: Physical, vol. 221, pp. $1-8,2015$. [Online]. Available: http://www.sciencedirect.com/science/article/pii/S0924424714004324

[12] C. D. Bellicoso, F. Jenelten, C. Gehring, and M. Hutter, "Dynamic locomotion through online nonlinear motion optimization for quadrupedal robots," IEEE Robotics and Automation Letters, vol. 3, no. 3, pp. 2261-2268, July 2018.

[13] P. Sardain and G. Bessonnet, "Forces acting on a biped robot. center of pressure-zero moment point," IEEE Transactions on Systems, Man, and Cybernetics - Part A: Systems and Humans, vol. 34, no. 5, pp. 630-637, Sep. 2004.

[14] C. D. Bellicoso, C. Gehring, J. Hwangbo, P. Fankhauser, and M. Hutter, "Perception-less terrain adaptation through whole body control and hierarchical optimization," in 2016 IEEE-RAS 16th International Conference on Humanoid Robots (Humanoids), Nov 2016, pp. 558564.

[15] M. Bloesch, C. Gehring, P. Fankhauser, M. Hutter, M. A. Hoepflinger, and R. Siegwart, "State estimation for legged robots on unstable and slippery terrain," in 2013 IEEE/RSJ International Conference on Intelligent Robots and Systems, Nov 2013, pp. 6058-6064.

[16] C. Gehring, C. D. Bellicoso, S. Coros, M. Bloesch, P. Fankhauser, M. Hutter, and R. Siegwart, "Dynamic trotting on slopes for quadrupedal robots," in 2015 IEEE/RSJ International Conference on Intelligent Robots and Systems (IROS), Sep. 2015, pp. 5129-5135.

[17] C. D. Bellicoso, F. Jenelten, P. Fankhauser, C. Gehring, J. Hwangbo, and M. Hutter, "Dynamic locomotion and whole-body control for quadrupedal robots," in 2017 IEEE/RSJ International Conference on Intelligent Robots and Systems (IROS), Sep. 2017, pp. 3359-3365.

[18] C. Gehring, S. Coros, M. Hutler, C. D. Bellicoso, H. Heijnen, R. Diethelm, M. Bloesch, P. Fankhauser, J. Hwangbo, M. Hoepflinger, and R. Siegwart, "Practice makes perfect: An optimization-based approach to controlling agile motions for a quadruped robot," IEEE Robotics Automation Magazine, vol. 23, no. 1, pp. 34-43, March 2016.

[19] M. Hutter, C. Gehring, D. Jud, A. Lauber, C. D. Bellicoso, V. Tsounis, J. Hwangbo, K. Bodie, P. Fankhauser, M. Bloesch, R. Diethelm, S. Bachmann, A. Melzer, and M. Hoepflinger, "Anymal - a highly mobile and dynamic quadrupedal robot," in 2016 IEEE/RSJ International Conference on Intelligent Robots and Systems (IROS), Oct 2016, pp. $38-44$. 